\title{
The persistent hypoglossal artery
}

\author{
J. G. BLAIN ${ }^{1}$ AND J. LOGOTHETIS \\ From the Division of Neurology, University of Minnesota Hospitals, \\ University of Minnesota, U.S.A.
}

Persistence of primitive intracranial arteries in the adult is rare but the advent of angiography has made the demonstration of such anomalies more frequent. On occasion such persistent vessels were held to be responsible for the production of neurological deficit (Sutton, 1950; Jackson, 1964). Persistence of the hypoglossal artery has been demonstrated in only four pathological specimens (Batujeff, 1889; Oertel, 1922; Sutton, 1950; Morris, and Moffat, 1956). More recently it has been described angiographically in four patients (Begg, 1961 ; Bruetman, and Fields, 1963; Gerlach, Jensen, Spuler, and Viehweger, 1963; Jackson, 1964). The following report concerns another case of persistence of the hypoglossal artery, a review of the literature, and a discussion on the pathogenesis of the clinical picture associated with such lesions.

\section{CASE REPORT}

M.A., a 46-year-old white woman, was admitted to the University of Minnesota Hospitals on 9 September 1963 following a generalized motor seizure one week before admission.

Her past history was unrevealing except for an adenocarcinoma of the right breast treated in October 1959 by radical mastectomy, radiation therapy, and bilateral salpingo-oopherectomy.

Physical examination was normal except for the mastectomy scar and a mild increase in stretch reflexes in the left extremities.

Laboratory tests revealed a normal haemogram; urine analysis, blood urea nitrogen, fasting and two-hour post-prandial blood sugar levels, blood Kline test, serum electrolytes, alkaline phosphatase, and bromsulphthalein retention were also normal. Erythrocyte sedimentation rate was $25 \mathrm{~mm}$. in one hour. A mercury 203 brain scan showed no focal uptake. Chest and skull radiographs showed no abnormalities. The electroencephalogram was mildly abnormal because of low voltage slowing over the right hemisphere, most evident posteriorly.

During the patient's stay in hospital, two seizures were observed, one of which began with adversive movements of the head and eyes to the left as well as chewing movements of the mouth. A persistent left Babinski sign was elicited following the second seizure.

Bilateral carotid angiography was carried out by

'Present address: New York University Medical Center, 550 First Avenue, New York, New York 10016. percutaneous puncture of the common carotid artery. All film studies were made using the Amplatz seriograph and automatic injection syringe with programme selector (Amplatz, 1960, 1963) injecting $12 \mathrm{ml}$. of 50\% diatrazoate sodium (Hypaque) per injection. Antero-posterior and lateral projections of the left and the right carotid circulations were done using one injection per projection. An additional injection of the right carotid artery was done to visualize its extracranial portion. The extracranial and intracranial circulation on the left side was normal. However, on the right side, a large vessel was found originating from the internal carotid artery $3 \mathrm{~cm}$. above the bifurcation. The external carotid artery was not visualized distal to the common carotid artery bifurcation. The abnormal vessel coursed posteriorly and cephalad, entered the skull and joined the basilar artery, which was clearly visualized along with its major branches. This anomalous vessel equalled in size the internal carotid artery itself. The basilar artery was displaced to the right at its caudal origin and appeared to be receiving most of its blood supply from the anomalous vessel (Figs. 1, 2).

During the third injection on the right side, the patient experienced nausea and blurred vision which cleared promptly. On returning to the ward she was conscious. One hour later she had an adversive seizure with head and eyes turning to the left and generalized clonic movements. In the immediate post-ictal period there was confusion that persisted for one hour and examination at that time revealed a definite left-sided weakness with hypaesthesia and autotopagnosia on the left side. Subsequently, the patient became lethargic, developed weakness of the muscles innervated by the ninth and tenth cranial nerves, and the right pupil was found to be enlarged but reactive to light and accommodation.

During this time, the patient received diphenylhydantoin (Dilantin), $100 \mathrm{mg}$. three times daily, phenobarbital, $60 \mathrm{mg}$. four time daily, and dexamethasone (Decadron), $2 \mathrm{mg}$. four times daily. On this regimen she slowly improved and within a few days was able to feed herself. She was discharged much improved 10 days following angiography. Examination on discharge revealed mild lethargy, minimal weakness of the muscles of deglutition, a left hemiparesis with hyperreflexia, and a positive Babinski reponse. We learned two months later from her own physician that these difficulties were persisting though much improved.

Although the studies revealed no mass, it was felt that the patient in all probability still has a metastatic carcinoma to the cerebrum from a primary carcinoma of the breast. 


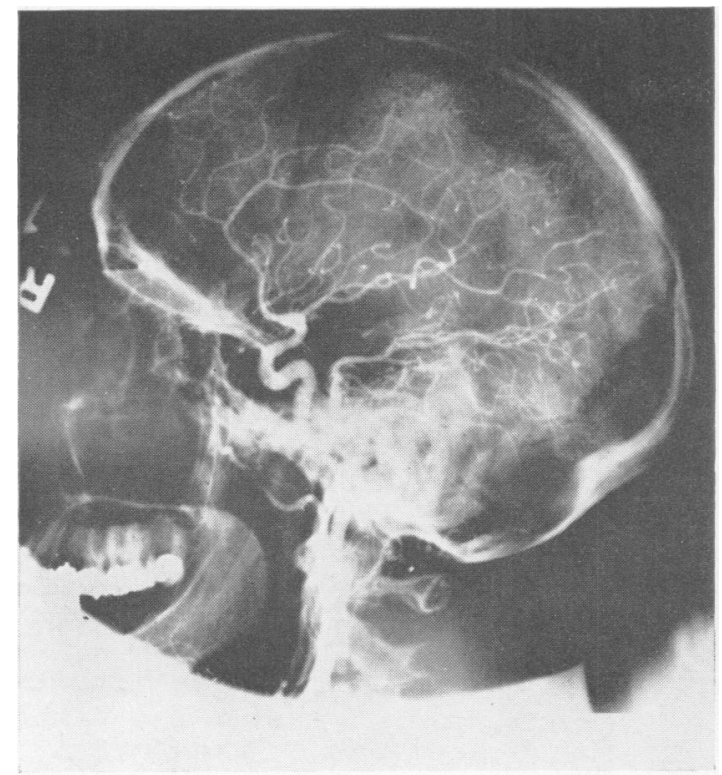

FIG. 1a

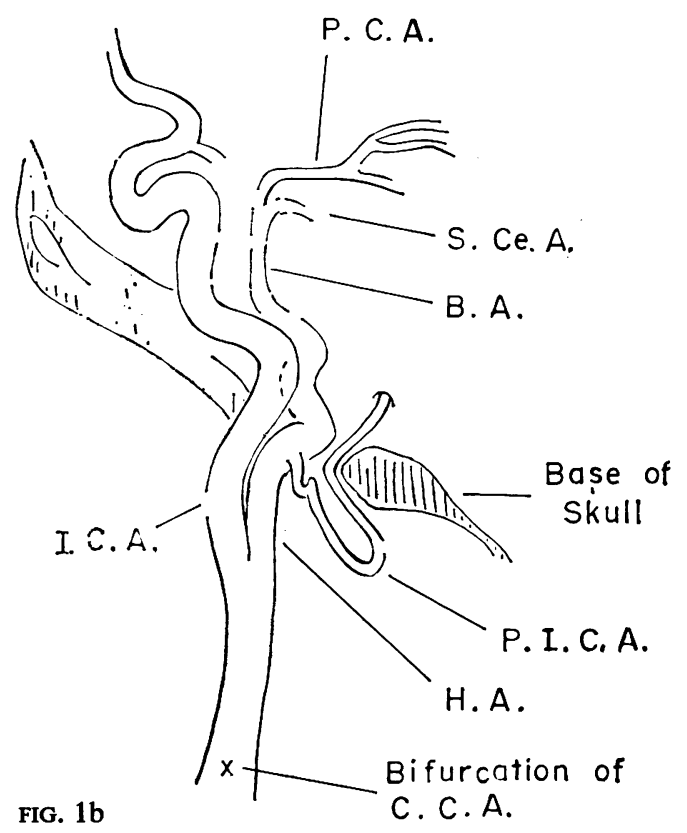

FIG. 1. Lateral projection of the arterial phase after injection of the right common carotid artery with diatrazoate sodium and schematic representation. The common carotid artery bifurcation is not visualized. The hypoglossal artery (H.A.) arises from the internal carotid artery (I.C.A.) at the level of the spinous process of C.2. The posterior inferior cerebellar artery (P.I.C.A.) arises from the hypoglossal artery. The posterior cerebral artery (P.C.A.) and superior cerebellar artery (S.Ce.A.) are visualized. Other vessels include the basilar artery (B.A.); note the bifurcation of the common carotid artery (C.C.A.).

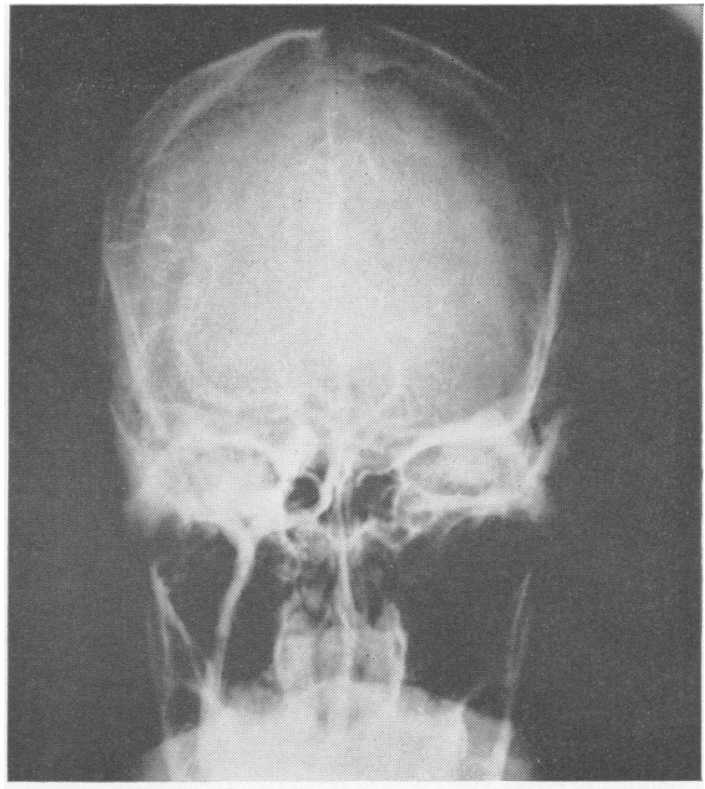

FIG. $2 \mathrm{a}$

A. Ch. A.

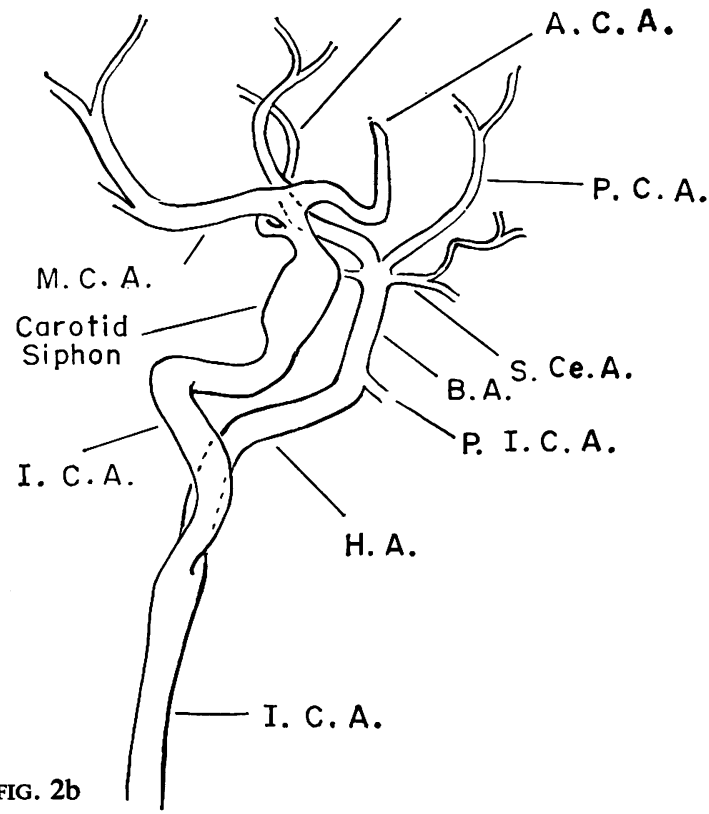

FIG. 2. Anterior posterior projection and schematic representation. The basilar artery (B.A.) is displaced to the right of the midline. The early course of the hypoglossal artery (H.A.) is parallel to that of the internal carotid artery (I.C.A.) then shifts medially to enter the intracranial cavity through the hypoglossal foramen and supply the basilar artery. The intracranial vessels otherwise have a normal appearance. A.Ch.A. anterior choroidal artery: A.C.A., anterior communicating artery: M.C.A., middle cerebral artery: P.C.A., posterior communicating artery: P.I.C.A., posterior interior cerebellar artery: S.Ce.A., superior cerebellar artery. 


\section{REVIEW OF EMBRYOLOGY AND ANATOMY}

In the $4 \mathrm{~mm}$. embryo the dorsal aorta, the rostral portion of which gives rise to the internal carotid artery, is well developed.

The basilar artery forms from the fusion of the two longitudinal neural arteries, which, in the $4 \mathrm{~mm}$. embryo, lie on either side of an avascular strip on the ventral aspect of the brain-stem (Congdon, 1922; Padget, 1948). The fusion is usually completed by the $8 \mathrm{~mm}$. stage. Failure of this fusion results in duplication of the basilar artery (Congdon, 1922). Lateral to each longitudinal neural artery is the primitive lateral basilo-vertebral anastomosis of Padget (Padget, 1948), and from it originate many vessels which form a plexiform network with the longitudinal neural artery.

In the cervical area, the intersegmental arteries arise from the dorsal aorta and accompany the cervical nerve roots. Cephalad to the first cervical intersegmental artery, corresponding to the second cervical root, are four recognized pairs of segmental arteries.

The first and most anterior pair is the trigeminal artery which connects the rostral portion of the dorsal aorta to the developing basilar artery. The trigeminal artery usually involutes by the $12 \mathrm{~mm}$. stage, by

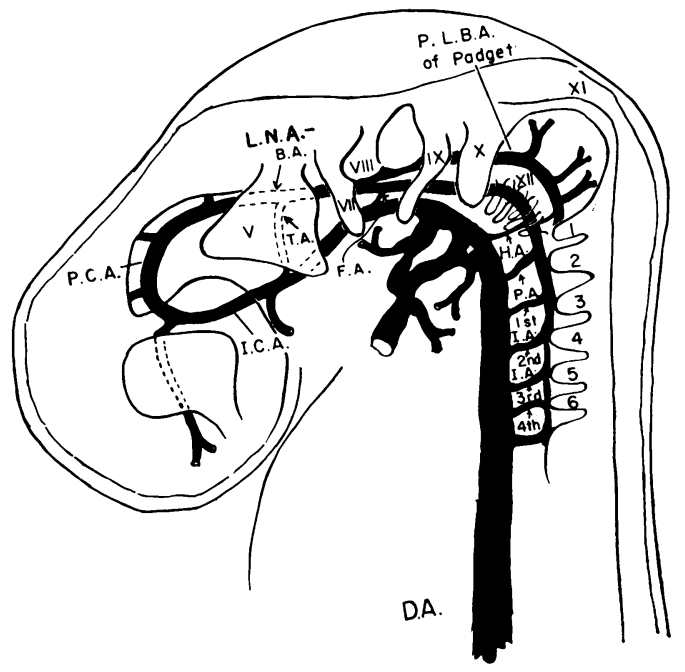

FIG. 3. Composite diagram (after Padget) of the $4 \mathrm{~mm}$. embryo illustrating the relative relationships of the vascular development: I.C.A., internal carotid artery: P.C.A., posterior communicating artery: L.N.A.-B.A., longitudinal neural artery or basilar artery: T.A., trigeminal artery: F.A., facio-acoustic artery: P.L.B.A., of Padget, primitive lateral basilo-vertebral anastomosis of Padget: H.A., hypoglossal artery: P.A., pro-atlantal artery: I.A., intersegmental artery: D.A., dorsal aorta (carotid artery). which time the posterior communicating artery is well developed (Padget, 1948).

The second pair, the facio-acoustic artery, lies at the level of the acoustic nerve.

The third pair joins the dorsal aorta to the longitudinal neural artery and the primitive lateral basilo-vertebral anastomosis of Padget in the region of the hypoglossal nerve rootlets. This is the hypoglossal artery. Schmeidel has referred to this vessel as a complex of two or three arteries (Schmeidel, 1932) whereas Padget refers to it as one vessel (Dandy, 1944; Padget, 1948).

The hypoglossal artery normally regresses by the $8 \mathrm{~mm}$. stage (Schmeidel, 1932) at which time the embryological basilar artery receives its main blood supply from the pro-atlantal artery which becomes correspondingly larger (Morris and Moffat, 1956), and represents the fourth pair of intersegmental arteries.

The vertebral artery develops during the 7 to $9 \mathrm{~mm}$. stage by fusion of the cervical intersegmental arteries

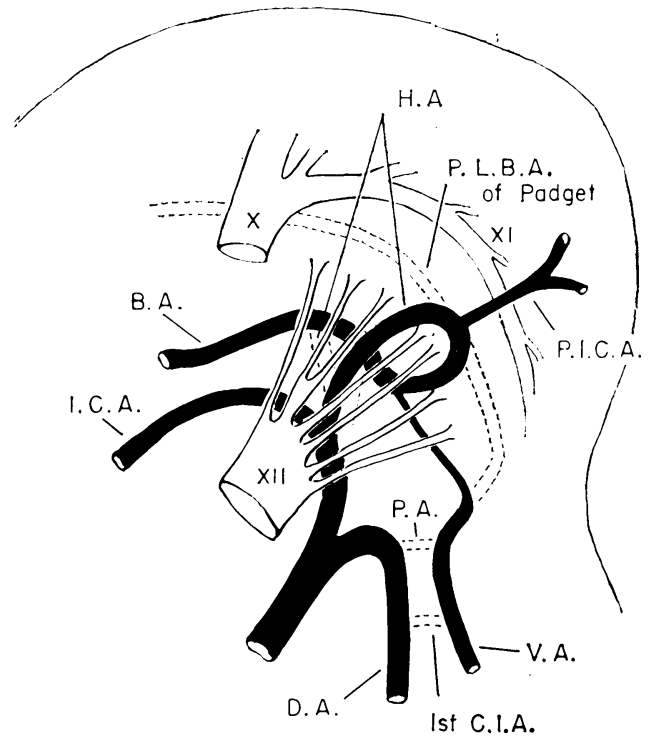

FIG. 4. Diagram illustrating the shift in course of the hypoglossal artery (H.A.) in its development. The embryological vessel involutes at its basilar portion (broken lines) whereas the anastomotic portion to the primitive lateral basilo-vertebral anastomosis of Padget (P.L.B.A. of Padget) enlarges to become the anomalous vessel in the adult. This explains the position of the persistent vessel in the adult. Note the origin of the posterior inferior cerebellar artery (P.I.C.A.) at the point where the primitive hypoglossal artery joins the primitive lateral basilovertebral anastomosis of Padget. I.C.A., internal carotid artery: B.A., basilar artery: P.A. pro-atlantal artery: V.A., vertebral artery: 1 st C.I.A., first cervical intersegmental artery: D.A., dorsal aorta. 
at their aortic ends to form a single tortuous vessel, starting from the sixth intersegmental artery and proceeding cephalad. The vertebral artery is completed by fusion with the pro-atlantal artery rostrally and with the seventh cervical intersegmental artery caudally which is to become the future subclavian artery (Congdon, 1922; Dandy, 1944; Padget, 1948).

In the pathological demonstration of the hypoglossal artery (Fig. 4), the anomalous vessel lies lateral and dorsal to the hypoglossal nerve rootlets in contrast to the embryological state where it lies medial and ventral to the nerve rootlets (Batujeff, 1889; Begg, 1961; Moffat, 1962; Morris and Moffat, 1956). Morris and Moffat postulated on this basis that the anomaly in the adult is not due to persistence of the embryological hypoglossal artery per se, but is the result of the persistence of one of the anastomotic channels running from the carotid portion of the hypoglossal artery to the lateral basilovertebral anastomosis of Padget, and in turn communicating with the basilar artery (Fig. 4) (Morris and Moffat, 1956).

\section{COMMENT}

The clinical significance of this anomaly is as yet unclear. Since 1961, angiographic demonstration of the persistent hypoglossal artery has been described in four patients. Bruetman and Fields (1963) were unable to demonstrate a vertebral artery on the affected side and noted that the vessel on the contralateral side was hypoplastic. The presence of this vessel has been associated with syncope on head turning in a young patient (Jackson, 1964). Its clinical significance seems clear in the patient of Gerlach et al. (1963), where recognition of the anomaly before repair of an arteriovenous fistula in the neck prevented a possible death as the result of basilar artery insufficiency.

Begg (1961) has alluded to the possible consequence of arteriosclerotic occlusion of the carotid artery on the affected side, though no such cases are reported in the literature. The presence of the persisting hypoglossal artery does not adequately explain the recent onset of seizures in our patient. These are more readily explained by the presence of cortical or meningeal implants of metastatic tumour. However, the post-angiographic course of the patient can partially be attributed to the presence of the anomaly, stupcr, parietal lobe findings, seizures, hemiparesis, and anisocoria suggesting cortical dysfunction and impending herniation. The presence of dysphagia and dysarthria points to a brain-stem dysfunction. The time of onset of these findings is compatible with the onset of oedema associated with injection of diatrazoate sodium intra-arterially (Hammergran, Geise, and
French, 1964). The improvement following the use of dexamethasone would further support this hypothesis (Galicich, French, and Melby, 1961).

Early recognition of this anomaly, therefore, is important, as repeated injections of concentrated dye into the carotid and basilar arteries simultaneously, because of the presence of this anomalous vessel, which may be the sole contributing artery to the basilar artery, may precipitate symptoms of a transient or permanent nature.

\section{SUMMARY}

A case of persistence of the hypoglossal artery is reported which was found incidentally in the course of an angiographic study of a patient who had had a seizure. The embryology of this anomaly is briefly reviewed, with particular reference to a possible causal association with the failure of development of the vertebral arteries. The literature of the previously reported cases is reviewed.

The authors are indebted to Dr. A. C. Begg and the publishers of Clinical Radiology for permission to reproduce Figures 3 and 4.

\section{REFERENCES}

Amplatz, K. (1960). A vascular injector and program selector. Radiology, 75, 955-956.

(1963). Automatic injection syringe and cassette changer for cerebral angiography. J. Amer. med. Ass., 183, 430-433.

Batujeff, N. (1889). Eine Seltene Arterienanomalie (Ursprung der A. Basilaris aus der A. carotis interna). Anat. Anz., 4, 282-285.

Begg, A. C. (1961). Radiographic demonstration of the 'hypoglossal artery'. A rare type of persistent anomalous carotid-basilar anastomosis. Clin. Radiol., 12, 187-189.

Bruetman, M. E., and Fields, W. S. (1963). Persistent hypoglossal artery. Arch. Neurol. (Chic.), 8, 369-372.

Congdon, E. D. (1922). Transformation of the aortic-arch system during the development of the human embryo. Contr. Embryol. Carneg. Instn, 14, 47-110 [No. 68].

Galicich, J. H., French, L. A., and Melby, J. C. (1961). Use of dexamethasone in treatment of cerebral edema associated with brain tumors. $J$-. Lancet, 81, 46-53.

Gerlach, J., Jensen, H. P., Spuler, H., and Viehweger, G. (1963) Traumatic carotico-cavernous fistula combined with persisting primitive hypoglossal artery. $J$. Neurosurg., 20, 885-887.

Hammergran, L. L., Geise, A. W., and French, L. A. (1964). Protection against cerebral damage from intracarotid injection of hypaque. Univ. Minn. med. Bull., 30, 374-376.

Jackson, F. E. (1964). Syncope associated with persistent hypoglossal artery. Case report. Ibid., 21, 139-141.

Moffat, D. B. (1962). The embryology of the arteries of the brain. Ann. roy. Coll. Surg. Engl., 30, 368-382.

Morris, E. D., and Moffat, D. B. (1956). Abnormal origin of the basilar artery from the cervical part of the internal carotid and its embryological significance. Anat. Rec., 125, 701-711.

Oertel, O. (1922). Uber die Persistenz embryonaler Verbindungen zwischen der Arteria carotis interna und der Arteria vertebralis cerebralis. Anat. Anz., 55, suppl., pp. 281-291. [Verh. anat. Ges.].

Padget, D. H. (1944). In Intracranial Arterial Aneurysms, by W. E. Dandy, p. 67. Comstock, Ithaca, New York.

(1948). The development of the tranial arteries in the human embryo. Contr. Embryol. Carneg. Instn, 32, 205-261.

Schmeidel, G. (1932). Die Entwicklung der a. vertebralis beim Menschen. Morph.Jb., 71, 315-335.

Sutton, D. (1950). Anomalous carotid-basilar anastamosis. Brit. J. Radiol., 23, 617-619. 